\title{
Exploración de la intensidad motivacional para la búsqueda del sentido de la vida en estudiantes universitarios de psicología*
}

\author{
José Antonio Cuny
}

El presente estudio tiene como finalidad explorar la intensidad motivacional para la búsqueda del sentido de la vida, a la luz de la logoterapia,en estudiantes universitarios de psicología de una universidad privada de Lima. La muestra estuvo conformada por 100 alumnos, cuyas edades fluctúan entre los 18 y 26 años. Se utilizaron tres instrumentos: el Test de Búsqueda de Metas Noéticas (Seeking for Noetic Goal Test) SONG, un cuestionario de indicadores sociodemográficos y un cuestionario autobiográfico. El resultado más destacable es que existen diferencias significativas en los puntajes obtenidos por los hijos de padres que viven separados y de padres fallecidos en comparación con los alumnos que tienen padres que viven juntos. En la parte cualitativa los jóvenes manifiestan tener temor a la muerte, la soledad y el futuro.

Logoterapia / intensidad motivacional / metas espirituales

Study of motivational intensity in the search for the meaning of life in psychology majors

This study aims at exploring motivational intensity in the search for the meaning of life, from a logotherapy perspective, in psychology students in a private university in Lima. The sample consisted of 100 students, ages from 18 to 26 years. Three instruments were utilized: Seeking Noetic Goal Test (SONG), a questionnaire of sociodemographic indicators and an autobiographical questionnaire. There were significant differences in scores of children of separated parents and deceased parents in comparison to students who live with both parents. In the qualitative assessment, students showed fear to death, loneliness and their future.

Logotherapy /motivational intensity /spiritual goals

* Estudio auspiciado por el Instituto de Investigación Científica de la Universidad de Lima. 
La logoterapia es un tipo de psicoterapia que tiene entre otras influencias la del existencialismo. Este es un movimiento en filosofía que data de la primera mitad del siglo XIX, cuando Soren Kierkergaard (1813-1855) comenzó criticando la intelectualización y el racionalismo de la filosofía de Georg Wilhelm F. Hegel (1770-1831). Esta corriente filosófica trata algunas cuestiones importantes de la existencia humana, como la autenticidad, la voluntad de sentido y la búsqueda de significado en la vida, que permiten la conformación de un proyecto existencial. También Federico Nietzsche y Arthur Schopenhauer son filósofos que se ubican en el inicio de este movimiento. Posteriormente, Jean Paul Sartre, Simone de Beauvoir y Albert Camus desarrollan este movimiento existencial tanto en filosofía como en literatura en el siglo XX.

Es importante desarrollar la capacidad de reflexión para ser autónomos en la proyección de nuestra vida, en la posibilidad de vivir una existencia basada en nuestras verdaderas metas, tanto en la dimensión personal como en la dimensión social. Una sensación de falta de sentido en el futuro, trae consigo una falta de respuesta en el presente

Estos temas deben ser vistos a la luz del desarrollo humano, ya que son preocupaciones que son vistas con diferente óptica en la juventud, en la adultez o en la vejez.
Los psicólogos del desarrollo (Baltes, 1987) tienen la preocupación de responder preguntas como: ¿Qué va sucediendo en estos períodos? ¿Qué nuevos datos puede aportar la psicología para comprender mejor estos procesos personales? ¿Se puede intentar cuantificar lo holístico?

¿Qué es aquello que nos impulsa en la vida? ¿Cuál es la motivación que acompaña a los jóvenes a realizar sus estudios? No es sencillo definir motivación. En general es un término referido a deseo, aspiración, impulso que tiene un organismo; lograr un fin u obtener una satisfacción. Existen interpretaciones teleológicas y no teleológicas de la motivación.

Metas noéticas significa la búsqueda de planteamientos espirituales. Intentar responder a preguntas como: ¿Qué buscan los jóvenes que les dé significado a sus vidas?, o, por el contrario, ¿están evadiendo su búsqueda y su responsabilidad existencial?

El estar en el mundo demanda una acción personal para crecer y desarrollarse psicológicamente. Requiere esfuerzo, decisión y selección de alternativas, que eventualmente pueden no ser populares. El grupo no puede suplantarnos en nuestra autenticidad.

La persona, entonces, tiene que definir dos proyectos existenciales: 1) un proyecto profesional acompañado de su dimensión social, y 2) la parte individual y la construcción personal. Es 
importante que estos dos proyectos sean paralelos y que se complementen uno al lado del otro.

\section{ANÁLISIS EXISTENCIAL}

El análisis existencial es la aplicación de la filosofía existencial a la psicología, a la teoría de la personalidad y a la psicoterapia. El término Dasein se refiere a estar en el mundo, el mero estar en el mundo. Martín Heidegger (1950) pensaba que la existencia es el existente, que somos nosotros mismos; el modo de ser en el que uno puede relacionarse consigo mismo, puede comprenderse a sí mismo. Para Karl Jaspers (1967) tener existencia es saber que soy un ser viviente con principio y fin, con espacio de realidad en el que está todo lo que soy y lo que es para mí. En forma general, se puede decir que el análisis existencial es el nombre filosófico de la psicología y de la psicoterapia existencial.

Dos autores han tenido el liderazgo en realizar esta aplicación de la filosofía a la psicología, ambos médicos suizos y admiradores de M. Heidegger, en visión filosófica, e inicialmente entrenados en psicoanálisis clásico (freudiano y jungiano): Ludwing Binswager (1881-1966) y Medard Boss (19031990).

Fue Binswanger, bajo el influjo de Edmund Husserl (1859-1938), Max Scheler (1874-1928), y sobre todo de la fenomenología de Martín Heidegger
(1889-1976), quien aplicó y modificó algunos conceptos del análisis existencial. A diferencia de la ontología fundamental de Heidegger, que buscó analizar la pregunta por el sentido del ser, a Binswanger le interesó analizar empíricamente las diversas maneras de existir, partiendo de la analítica existencial (Daseinsanalytik) de Heidegger, con el fin de darle fundamento filosófico a una antropología empírica.

A diferencia de las investigaciones psicoanalíticas o de las que se orientan en las ciencias naturales, basadas ambas en la causalidad, el análisis existencial intenta encontrar un nivel de experiencia y de interpretación más profundo. No pretende ir más allá de los fenómenos a fin de buscar su explicación (causal), sino que busca analizar su sentido partiendo de ellos mismos.

El paso del análisis estructural $a$ priori al análisis empírico, lo indicó Binswanger con el término Daseinsanalyse (análisis existencial), a diferencia del término Daseinsanalytik (analítica existencial) utilizado por Heidegger. Con este término se separó Binswanger también del psicoanálisis (Psychoanalyse) de Sigmund Freud (1856-1939).

La crítica existencial al concepto freudiano de inconsciente, así como una concepción mecanicista y naturalista de la psyche, es también uno de los puntos centrales del segundo fundador del análisis existencial como escuela terapéutica, Medard Boss, discí- 
pulo de Freud y asistente de Eugen Bleuler (1857-1939), estudió en Berlín y Londres, donde pertenecía al círculo de la neopsicoanalista Karen Horney. Toma contacto con Carl G. Jung en la década de 1930. Pero es el encuentro personal con Martín Heidegger, en 1946, que deviene decisivo para su pensamiento.

Boss basa su posición fenomenológica en una crítica a las posiciones de tipo positivista o naturalista, que intentan explicar fenómenos psíquicos, ya sea sobre la base de un reduccionismo físico o uno biológico. En el nuevo fundamento que Boss expone se trata, en primer lugar, de mostrar fenomenológicamente algunas de las dimensiones del existir humano en su especificidad y sin pretender explicarlas o demostrarlas partiendo de otros fenómenos aparentemente fundamentales. Este método, que Boss toma de Heidegger (y este a su vez de Husserl), es el que está en la base de su interpretación de las neurosis, de los sueños y con ello de la práctica misma del análisis existencial.

\section{LOGOTERAPIA Y ANÁLISIS EXISTENCIAL}

Tejer tenues hebras de vidas rotas en una urdimbre firme, coherente, significativa y responsable es el objeto con que se enfrenta la logoterapia, que es la versión original de Viktor Frankl (1905-1997) del moderno análisis existencial. Frankl (2001) afirmó que la búsqueda por parte del hombre del sen- tido de la vida constituye una fuerza primaria y no una "racionalización secundaria" de sus impulsos instintivos. Esta búsqueda de sentido es única y específica en cuanto que es uno mismo quien tiene que encontrarlo; únicamente así logrará alcanzar el hombre un significado que satisfaga su propia voluntad de sentido.

La logoterapia y el análisis existencial son las dos caras de una misma teoría. Es decir, la logoterapia es un método de tratamiento psicoterapéutico, mientras que el análisis existencial representa una orientación antropológica de investigación.

En logoterapia, "logos" quiere decir dos cosas: por un lado, el sentido y, por el otro, lo mental.

Según el análisis existencial no existe solamente el carácter instintivo inconsciente, sino también el carácter mental inconsciente; dicho en otras palabras: no solo conocemos y reconocemos un inconsciente instintivo sino también un inconsciente mental y el logos, al que la conformación terapéutica del análisis existencial, la logoterapia, convierte tanto en el hacia dónde y en el de dónde de sus esfuerzos, tiene sus raíces en el inconsciente.

En el análisis existencial, existencial significa una forma de ser y en especial el carácter propio del hombre. Para esta forma especial de la existencia (Dasein) la filosofía contemporánea reserva la expresión existencia 
(Existenz), y en el análisis y la logoterapia se ha tomado prestada esta expresión con este contenido.

El análisis existencial es una explicación de la existencia y no un análisis o una síntesis de esta. La existencia, la persona, también se explica a sí misma: se explica, se despliega, se desarrolla en el transcurso de la vida. Es una explicación ontológica de lo que es la existencia. En este sentido, el análisis existencial constituye el intento de una antropología psicoterapéutica, de una antropología que precede a cualquier psicoterapia, no solo a la logoterapia, pues el análisis existencial es el fundamento intelectual imprescindible de la psicoterapia.

La logoterapia y el análisis existencial tienen cinco aspectos, que son los siguientes:

- Análisis existencial como explicación de la existencia personal.

- Análisis existencial como terapia de neurosis colectivas.

- Análisis existencial como cura de almas médicas.

- Logoterapia como terapia específica de neurosis noógenas.

- Logoterapia como terapia no específica.

A pesar de que la logoterapia y el análisis existencial parten de la praxis clínica no se puede evitar que desemboquen en una teoría que va más allá de la clínica.
El objetivo del análisis existencial como explicación antropológica de la existencia personal consiste en hacer consciente, explicar, desplegar y en desarrollar la concepción implícita, inconsciente, que la persona tiene de sí misma.

El análisis existencial caracteriza y califica la esencia de la existencia en el sentido de que la existencia es una forma de ser, a saber, el ser humano, el ser específico del hombre, cuya particularidad consiste en que por lo que atañe al hombre no se trata de un ser de hecho sino de un ser facultativo, no de un deber ser así y no de otra forma $-\mathrm{y}$ en este sentido el hombre neurótico entiende mal su propia esencia-, sino más bien de un poder llegar a ser también de otra forma.

\section{LA DIMENSIÓN ESPIRITUAL: LO NOÉTICO}

De acuerdo con la logoterapia, la primera fuerza motivante del hombre es la lucha por encontrarle sentido a la propia vida, a la vida tal cual es, y toda vida por más adversa que sea siempre tiene algún sentido.

Pero el sentido de la vida no puede ser dado sino que debe ser hallado por uno mismo.

Por eso, a pesar de los problemas que podamos tener, toda vida vale la pena ser vivida y más aún cuando el hombre pone en práctica la fuerza de oposición del espíritu frente al destino, o sea frente a aquello con lo que me en- 
cuentro en la vida sin haberlo elegido; pero ante lo cual sigo siendo libre de actuar, de un modo o de otro.

El sentido está siempre cambiando, pero jamás falta.

En caso de no verlo, habrá que dotar a la vida de sentido aun en las situaciones más difíciles, donde lo que importa es dar testimonio de la mejor y exclusiva potencialidad humana: la de transformar la tragedia, la enfermedad y el fracaso en un triunfo personal, en un logro humano.

La logoterapia despierta a la persona en su rol de protagonista de su propia historia, de su felicidad, de sus logros y en su rol de constructor de su persona dado que el hombre es un ser llamado a elegir un proyecto de vida en conformidad con su propio ser, por lo tanto artífice de su destino.

Proyecto de vida no son las ocurrencias antojadizas con las que llenamos el tiempo de la vida, sino la orientación organizada de los esfuerzos para darle más sentido a la vida.

La propuesta logoterapéutica es desarrollar la actitud de búsqueda de los para qué de las situaciones tanto del fracaso como del éxito, de visualizar el futuro no como una utopía o como algo que hay que saltear velozmente, sino como una posibilidad esperanzadora, la de asumir el compromiso de la búsqueda de la misión en la vida.
El hombre solo llega a ser tal en la medida en que descubre el sentido de la vida, el por qué y el para qué existir.

Frankl también habla del suprasentido, que es trascendente al hombre y es el sentido que excede la capacidad intelectual del hombre; accedemos a él por la fe, que es una fuerza terapéutica y propiciatoria del desarrollo humano. Esta fe fortalece al ser humano y hace que en el fondo nada carezca de sentido ni nada suceda en vano. Es una dimensión religiosa.

La logoterapia le da sentido a la vida, estimula la libertad responsable, la práctica de valores, autotrascendencia y sobre todo el espíritu de renuncia, de sacrificio.

Es el método de tratamiento psicoterapéutico que parte del espíritu y está centrado en la búsqueda de sentido. La logoterapia parte desde la dimensión espiritual.

$\mathrm{Su}$ objetivo es conducir a la persona a la autodeterminación, sobre la base de la propia responsabilidad, y solidificar el sentido individual que lo lleve a causas a las cuales servir o a personas a quienes amar.

\section{INVESTIGACIONES ANTECEDENTES}

James Crumbaugh y colaboradores han hecho un gran trabajo al desarrollar y perfeccionar herramientas psicométricas para evaluar los principales conceptos frankleanos. Por razones de es- 
pacio no detallaremos toda su producción. Crumbaugh es el creador del test que ocupa esta investigación: el SONG; también del PIL: test de propósito en la vida.

El SONG (Seeking of Noetic Goal Test), Test de Búsqueda de Metas Noéticas, evalúa el grado en que las personas buscan sentido a la vida, la fuerza de la motivación que una persona tiene para buscar este sentido existencial. Si una persona no encuentra un propósito o sentido estará muy motivado a hacerlo. Si ya lo tiene, entonces tendrá una baja motivación. En la práctica se encuentra que usualmente las personas buscan sentido porque no lo tienen y quieren tenerlo. Si no se encuentra entonces habrá una frustración existencial. Las investigaciones de Crumbaugh se han realizado con diferentes muestras para probar su confiabilidad, validez y capacidad de predecir diferencias grupales de los tests PIL y SONG, y también la combinación de ambos.

Reker y Cousins (1979) administraron ambos tests a estudiantes para analizar la estructura factorial, la validez de constructo y la confiabilidad de esas escalas. Los resultados confirman la afirmación de Crumbauhg de que el SONG y el PIL son tests válidos y confiables y adecuados predictores de una terapia.

María Ángeles Noblejas de la Flor realizó un trabajo denominado "Evaluación de los abandonos en las primeras fases de un programa de tratamiento de drogadicción" (1998). El estudio se sitúa en una línea de validación de la logoterapia, de aportación de datos empíricos que muestran la existencia en el hombre de su voluntad de sentido. Ello lo hace poniendo a prueba, en este caso, la capacidad de algunos instrumentos logoterapéuticos de evaluación para ser predictores de los abandonos de un programa terapéutico educativo que trabaja con personas que tienen problemas de adicción a las drogas.

Las posibles explicaciones para este fenómeno podrían ser la dificultad para afrontar la dureza del programa, la necesidad de ver resultados muy inmediatos, la falta de una motivación profunda para salir de la droga, etcétera. Desde una perspectiva logoterapéutica intentó rastrear una dificultad no ya en contar con una vida significativa, pues como pusimos de manifiesto anteriormente, se encuentran en una situación de vacío existencial, sino en las posibilidades para encontrar ese sentido.

Por ello, incorporó en su investigación (además del test PIL y el logotest ya citados) el test SONG ( Crumbaugh, 1977), propuesto por su autor como complementario al test PIL y que trata de evaluar la necesidad que experimenta la persona de encontrar sentido en la vida (Noblejas, 2000).

\section{Propósito de ESTE ESTUdio}

Específicamente, el trabajo del psicólogo exige mucho de sí mismo, llegando 
en ocasiones a comprometer su vida personal. Aparte de conocimiento, técnica y habilidad, el psicólogo, sobre todo el clínico, juega un rol central en la clase especial de trabajo que realiza. El ser psicólogo y, más aún, psicoterapeuta exige gozar de una vida equilibrada (Corveleyn, 1998).

Desde el punto de vista de la psicología del desarrollo, entre los 17 y los 24 años se suceden cambios físicos, psicológicos y sociales, sufriendo también modificaciones las motivaciones, los intereses y las expectativas. Se puede cuestionar la elección de la profesión. Lo académico y lo intelectual se entremezclan con los afectos, las situaciones familiares cotidianas y los planteamientos frente a la vida. Hay un mundo interno y un mundo externo con el que se tienen que relacionar.

Este proceso puede ser más complejo en los estudiantes de psicología, quienes por las materias que estudian pueden replantearse sus expectativas, plantearse otras preguntas y otros temas, en relación con el contenido de dichas materias, que pueden estar describiendo mucho de lo que vivencian ellos mismos o las personas de su entorno.

El presente estudio busca responder parte de estas inquietudes, saber más acerca de la búsqueda de metas en este período, específicamente en los estudiantes de psicología. Si esta búsqueda cambia poco o mucho en esta etapa de la vida, ¿son sus metas diferentes en el tiempo?, ¿el quién soy estará más claro para ellos con el tiempo?, ¿la vida va teniendo más sentido para estos jóvenes a medida que van terminando sus estudios universitarios?

Hace un tiempo respondimos algunos interrogantes sobre la soledad (Cuny, 2001) en jóvenes universitarios. Vimos la importancia que tienen los amigos y los padres en estos procesos del desarrollo. Tenemos la impresión de que las respuestas necesitan de trabajos de largo aliento para conocer en profundidad a nuestros jóvenes estudiantes.

En la medida en que averigüemos más sobre este proceso existencial que venimos describiendo, podremos intentar dar respuesta a las preguntas antes planteadas y dar una mejor orientación a estos jóvenes hacia una visión más clara del sentido existencial que podrían darle a su vida.

\section{MÉTOdo}

\section{Diseño de investigación}

El presente estudio, de acuerdo con los objetivos planteados, se enmarca en los criterios exploratorio y descriptivocomparativo. Intentaremos hacer un tratamiento también de tipo cualitativo, enmarcando la investigación dentro de una tipología mixta (Hernández, 1998). 


\section{Participantes}

El universo de la investigación son 228 alumnos pertenecientes al total de la población de estudiantes de la Facultad de Psicología en el período académico 2005-I.

\section{Composición de la muestra}

La muestra, estadísticamente significativa para la presente investigación, estuvo conformada por 100 alumnos de ambos sexos, de los ciclos: tercero, quinto, sétimo, noveno, undécimo y duodécimo (internado), matriculados en el período académico 2005-I de una universidad particular. El $41 \%$ de ellos pertenecientes al tercer ciclo, $11 \%$ al quinto ciclo, $10 \%$ al sétimo ciclo, $13 \%$ al noveno ciclo y $25 \%$ pertenecientes al internado (véase tabla 1).

Tabla 1

Composición de la muestra por nivel de estudios

\begin{tabular}{lcc}
\hline Variable & Frecuencia & Porcentaje \\
\hline $3^{\circ}$ ciclo & 41 & 41.0 \\
$5^{\circ}$ ciclo & 11 & 11.0 \\
$7^{\circ}$ ciclo & 10 & 10.0 \\
$9^{\circ}$ ciclo & 13 & 13.0 \\
Internado & 25 & 25.0 \\
\hline \multicolumn{1}{c}{ Total } & 100 & 100.0 \\
\hline
\end{tabular}

Se aprecia que el $71 \%$ de la muestra pertenece al sexo femenino y el $29 \%$ al sexo masculino (véase tabla 2). Con relación a las edades, vemos que estas fluctúan entre 18 y 49 años. Con un mayor predominio $(67 \%)$ de aquellos que se ubican entre los 18 y los 22 , seguidos por los que se encuentran entre los 23 y los 26 años (25\%); el 6\% están entre los 27 y los 29; un 1\% de 31 años y $1 \%$ de 49 años (véase tabla 3 ).

\section{Tabla 2}

Composición de la muestra por sexo

\begin{tabular}{ccc}
\hline Variable & Frecuencia & Porcentaje \\
\hline Femenino & 71 & 71.0 \\
Masculino & 29 & 29.0 \\
\hline Total & 100 & 100.0 \\
\hline
\end{tabular}

Tabla 3

Composición de la muestra por edad

\begin{tabular}{ccc}
\hline Variable & Frecuencia & Porcentaje \\
\hline $18-22$ & 67 & 67.0 \\
$23-26$ & 25 & 25.0 \\
$27-29$ & 6 & 6.0 \\
31 & 1 & 1.0 \\
49 & 1 & 1.0 \\
\hline Total & 100 & 100.0 \\
\hline
\end{tabular}

Con respecto a las demás variables sociodemográficas consideradas en el estudio como estado civil, el $98 \%$ de estudiantes son solteros y el $2 \%$ están casados. Sobre pertenencia a una religión el $64 \%$ señala ser católico, el $25 \%$ no se identifica con ninguna religión y el 4,3\% dice pertenecer a una Iglesia cristiana. En cuanto a nivel socioeconómico, un grupo mayoritario reside en distritos clasificados dentro de los seg- 
mentos A y B (80\%). La mayor proporción de estudiantes procede de colegios religiosos $(63 \%)$ y el $35 \%$ de colegios laicos. El 52\% de los estudiantes terminó el colegio a la edad de 17 años, seguidos por aquellos que terminaron a los 16 años (41\%). La mayoría de estudiantes ingresó a la universidad a la edad de 17 años (48\%).

Observamos en la muestra que el $79 \%$ de los padres viven juntos, seguidos por un menor porcentaje (15\%) que viven separados. El nivel educativo de los padres es, en su mayoría, superior $(88 \%)$ y en igual porcentaje (5\%) están aquellos que tiene educación superior incompleta o instrucción secundaria. La principal ocupación de los padres es de gerente (14\%), seguidos por profesionales como ingenieros $(9 \%)$ y médicos $(8 \%)$. Un porcentaje reúne a los empresarios (6\%). El grupo mayoritario de las madres (74\%) tiene instrucción superior, y le sigue el grupo que tiene grado de instrucción secundaria $(17 \%)$. El mayor porcentaje de madres (36\%) tiene como ocupación su casa, seguido por los grupos que se desempeñan como docentes $(11 \%)$ y como secretarias (7\%). En cuanto al número de hermanos, el mayor porcentaje se encuentra entre aquellos que tienen de uno $(40 \%)$ a dos $(39 \%)$ hermanos. El $51 \%$ de la población evaluada es el hijo menor y el $31 \%$ el mayor.

La mayoría de estudiantes no tiene ningún hobby (29\%), 22\% se dedica al arte y $19 \%$ al deporte.

\section{Instrumentos}

En este estudio se utilizó el test SONG, de James Crumbaugh. Un segundo instrumento consideró algunos indicadores sociodemográficos.

El tercer instrumento, estrictamente cualitativo, es un cuestionario autobiográfico cuyas preguntas versan sobre metas, planes futuros y motivaciones de los jóvenes que nos ayudaron a conocer mejor los procesos de desarrollo y su búsqueda de metas.

El objetivo de la prueba de Búsqueda de Metas Noéticas, creada desde la orientación logoterapeútica, es medir la intensidad motivacional de una persona para encontrarle sentido a su vida. Adaptada a nuestro medio (Bartschi, 1999) se encontró que es una prueba con confiabilidad, validez predictiva y que presenta validez de constructo.

Está conformada por un total de 20 ítems. Las respuestas están dadas por una escala de Likert que va del 1 al 7: nunca, rara vez, ocasionalmente, a veces, con frecuencia, muy seguido y constantemente.

El sujeto tiene que marcar en la posibilidad que elige. La puntuación total se obtiene a partir de una suma aritmética. Las posibles puntuaciones oscilan entre 20 y 140.

Un puntaje alto en el test indica que el individuo presenta una alta motivación para encontrar un sentido en su vida. 
El proceso de adaptación de esta prueba en nuestro medio fue estudiado a partir de los resultados obtenidos en una muestra conformada por 467 alumnos universitarios de ambos sexos, encontrándose que tiene validez y confiabilidad.

\section{Procedimiento}

Seleccionada la muestra de estudiantes universitarios, tomándose en cuenta las características previamente establecidas, se procedió a la recolección de datos, aplicándose los tres instrumentos en forma simultánea y colectiva.

Esta recolección de datos estuvo a cargo de dos alumnos de ciclos superiores, previamente capacitados en la forma de aplicación de los instrumentos.

\section{Resultados}

Lo que nos motivó a realizar la investigación es el planteamiento que el desarrollo académico y el tener una vida dotada de sentido a través de la búsqueda de metas espirituales nos acerca a una vida más equilibrada, característica muy importante, sobre todo en la profesión del psicólogo por el tipo de labor que desempeña.

El valor obtenido en el coeficiente de confiabilidad de Cronbach (0.887) nos indica que existe una consistencia interna en la prueba aplicada (véase tabla 4).

La correlación de los ítems con la escala total indica una correlación de moderada a alta, exceptuando el ítem $18(0.037)$ en el que se da un valor muy bajo (véase tabla 4 ).

Tabla 4

Análisis de Ítems y confiabilidad del SONG

\begin{tabular}{|c|c|c|c|}
\hline Ítem & M. & D.E. & $\begin{array}{c}\text { Correlación } \\
\text { item-test } \\
\text { corregido }\end{array}$ \\
\hline 1. Yo pienso sobre el sentido de la vida. & 4.88 & 1.499 & .568 \\
\hline $\begin{array}{l}\text { 2. Yo siento que a pesar de estar destinado a lograr algo im- } \\
\text { portante en la vida, no puedo averiguar exactamente } \\
\text { qué es. }\end{array}$ & 3.39 & 1.595 & .658 \\
\hline $\begin{array}{l}\text { 3. Yo procuro tener nuevas actividades o áreas de interés, pe- } \\
\text { ro estas pronto pierden su atractivo. }\end{array}$ & 3.04 & 1.238 & .455 \\
\hline $\begin{array}{l}\text { 4. Yo siento que algo le falta a mi vida, pero no sé qué es. } \\
\text { 5. Yo me siento inquieto. }\end{array}$ & 3.18 & 1.417 & .530 \\
\hline 6. Yo siento que el mayor éxito de mi vida no lo he alcanzado. & 3.49 & 1.360 & .469 \\
\hline 7. Yo espero que ocurra algo muy emocionante en mi futuro. & 4.89 & 1.421 & .420 \\
\hline $\begin{array}{l}\text { 8. Yo sueño despierto, imaginando que encuentro un nuevo } \\
\text { lugar para mi vida y una nueva identidad. }\end{array}$ & 4.87 & 1.361 & .436 \\
\hline $\begin{array}{l}\text { 9. Yo siento la necesidad de encontrar un sentido y un propósi- } \\
\text { to real en mi vida. }\end{array}$ & 2.93 & 1.635 & .517 \\
\hline
\end{tabular}


Continuación

\begin{tabular}{|l|c|c|c|}
\hline \multicolumn{1}{|c|}{ Ítem } & M. & D.E. & Correlación \\
\hline $\begin{array}{l}\text { 11. Parece que yo cambio el objetivo principal de mi vida. } \\
\text { 12. El misterio de la vida me confunde e incomoda. }\end{array}$ & 2.69 & 1.383 & .475 \\
13. Yo siento la necesidad de encontrar un "nuevo horizonte en & 3.17 & 1.552 & .579 \\
$\quad$ mi vida". & 3.491 & .626 \\
14. Antes de terminar algo, yo comienzo con otras cosas. & 1.476 & .447 \\
$\begin{array}{l}\text { 15. Yo siento la necesidad de aventura y de "conquistar nuevos } \\
\text { mundos". }\end{array}$ & 3.90 & 1.648 & .521 \\
$\begin{array}{l}\text { 16. En mi vida he sentido una fuerte necesidad de encontrarme } \\
\text { a mí mismo. }\end{array}$ & 4.38 & 1.674 & .611 \\
$\begin{array}{l}\text { 17. En ocasiones he pensado que había encontrado lo que esta- } \\
\text { ba buscando en la vida, dándome cuenta después de que no } \\
\text { era así. }\end{array}$ & 2.89 & 1.286 & .356 \\
$\begin{array}{l}\text { 18. Yo he tenido siempre un objetivo poderoso hacia el cual mi } \\
\text { vida ha estado orientada. }\end{array}$ & 4.68 & 1.746 & .037 \\
$\begin{array}{l}\text { 19. Yo he sentido la falta de una labor que valga la pena realizar } \\
\text { en la vida. }\end{array}$ & 3.00 & 1.393 & .518 \\
20. Yo he sentido la necesidad de lograr algo fuera de lo común. & 4.36 & 1.767 & .630 \\
\hline
\end{tabular}

Alfa de Cronbach $=0.887$

$\mathrm{N}=100$

La prueba de bondad de ajuste definió una distribución normal de los puntajes de la muestra (véase gráfico 1).

\section{Grafico 1 \\ Distribución de los puntajes del test de SONG}

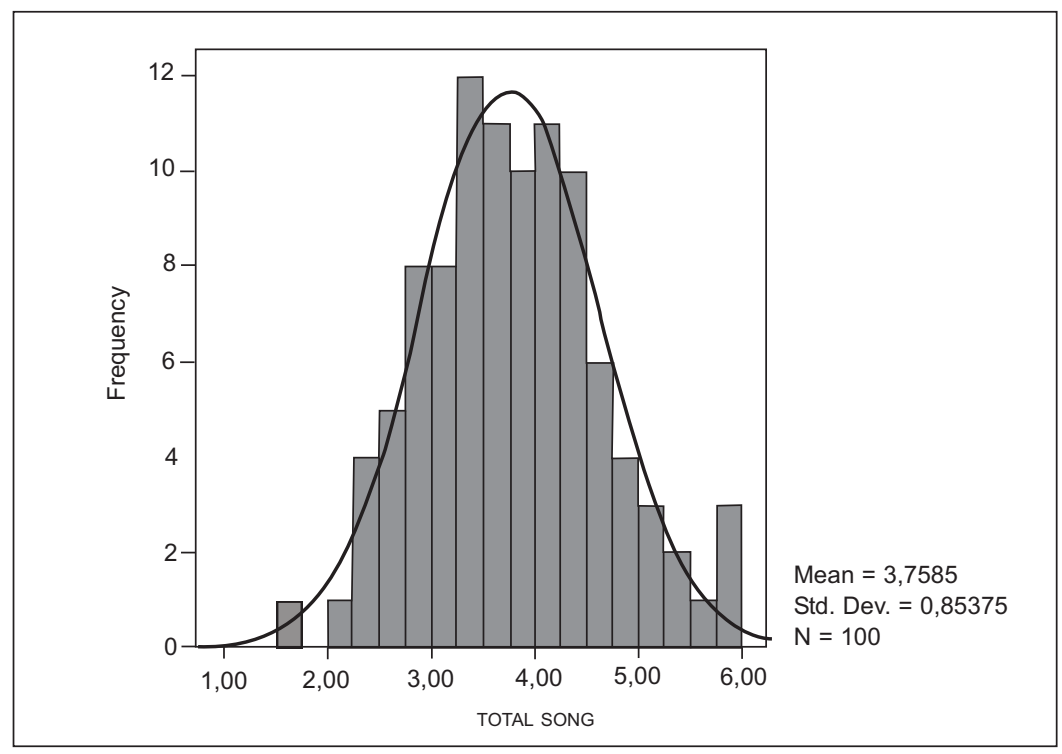


Las correlaciones más elevadas se encuentran en el ítem 2 (0.658), el ítem 9 (0.646), el ítem $20(0.630)$ y el ítem 13 (0.626), donde se observa la necesidad de buscar un propósito sin identificar aún cuál es, que es ratificada por el valor obtenido en el ítem 18, donde se señala puntualmente un objetivo.

En los resultados del análisis de correlaciones encontramos que no existen diferencias significativas en la comparación del SONG con las siguientes variables sociodemográficas: nivel de estudio, sexo, edad, Iglesia a la que pertenece, tipo de colegio de procedencia, número de hermanos, posición ordinal y si tiene o no un hobby.

Cuando el análisis correlacional se realiza con la variable sociodemográfica Relación entre los padres, se encuentra que existen diferencias significativas entre los puntajes de los sujetos con padres separados y los que tienen padres viviendo juntos, siendo los primeros los que obtienen puntajes más elevados en la prueba; es decir, el estudiante con padres separados posee una mayor intensidad motivacional para la búsqueda de metas noéticas (véase tabla 5), aunque aún no tienen claro qué es aquello que les falta, ya que dan puntajes altos en el ítem 4 (Yo siento que algo le falta a mi vida, pero no sé qué es).

También presentan diferencias significativas en los ítems 6, 9, 10, 13 y 15 , que expresan la necesidad de búsqueda de metas y una mayor intensidad motivacional.

Se obtuvieron valores significativos al correlacionar las preguntas 8 y 9 de los alumnos que tienen padres fallecidos con aquellos que tienen padres viviendo juntos, siendo los puntajes más altos los obtenidos por aquellos que tienen padres fallecidos. La pregunta 8 tiene que ver con la necesidad de encontrar un lugar para su vida y una nueva identidad y la 9 con la necesidad de encontrar un propósito en su vida.

En las preguntas 13 y 15 se dan diferencias significativas entre los puntajes (altos) obtenidos por los sujetos que tienen padres fallecidos y aquellos que tienen padres que viven juntos o padres que viven separados. Ambas preguntas tienen que ver con una necesidad de experimentar cambios en su vida (13. Yo siento la necesidad de encontrar un "nuevo horizonte en mi vida" y 15 . Yo siento la necesidad de aventura y de "conquistar nuevos mundos").

Tabla 5

Comparación del SONG por relación entre los padres

\begin{tabular}{lcccccc}
\hline Relación entre los padres & $\mathrm{N}$ & $\mathrm{M}$ & $\mathrm{D} . \mathrm{E}$. & $\mathrm{t}$ & $\mathrm{G} . \mathrm{L}$. & $\mathrm{P}$ \\
\hline Viven juntos & 79 & 3.6544 & .83171 & -2.638 & 92 & .10 \\
Separados & 15 & 4.2800 & .89678 & & & \\
\hline
\end{tabular}




\section{DISCUSIÓN}

Partiendo de un análisis cualitativo de los resultados por la información que aporta el cuestionario autobiográfico, se observa que los temas de mayor interés para los estudiantes son aquellos que tienen que ver con el área afectiva, la motivación vocacional y las actividades que realizan (hobbies, deportes, actividades culturales). En el tercero, quinto y sétimo ciclos existe una preocupación especial por las relaciones con sus coetáneos, necesidad de ayudar, autoconocimiento y bienestar general; centrándose la intensidad motivacional alrededor de estos temas, aunque no con una determinación clara de los medios y los objetivos. Con respecto al noveno ciclo y el internado se observa que existe una mayor claridad sobre qué medios utilizar para alcanzar sus objetivos (seguir una maestría, definición de área en la que se va a trabajar y proyectos personales: formar un instituto psicológico, fundar un hogar para niños, etcétera).

Dicha mayor claridad de este grupo en relación con sus compañeros de ciclos anteriores puede ser explicada como la plasmación de la búsqueda de metas espirituales para tener una vida más equilibrada y con mayor sentido.

Se halló una correspondencia entre las respuestas obtenidas en el cuestionario autobiográfico con los resultados cuantitativos obtenidos donde las correlaciones más elevadas se encuentran en los ítems donde se observa la necesidad de buscar un propósito sin identificar aún cuál es (ítems 2, 9, 20 y 13), que es ratificada por el valor obtenido en el ítem 18, donde se señala un objetivo, nos indica que progresivamente se van delineando los proyectos hasta convertirse en metas definidas que orientan su existencia.

Son los alumnos del quinto ciclo, en comparación con los del noveno ciclo y el internado, los que han obtenido las puntuaciones totales más elevadas en la prueba. Esto nos permite concluir que las personas que no tienen muy definido el propósito en su vida se encuentran más motivadas en buscar las metas noéticas que las que tienen más claridad y definición con respecto a sus objetivos. Cumbaugh plantea que si la persona siente que ha encontrado el sentido de su vida, presentará menor intensidad de búsqueda de metas noéticas.

Con respecto a las respuestas dadas por los alumnos del noveno ciclo se ha podido observar que en estos se presentan, en forma más recurrente, temas que tienen que ver con temor a la muerte de seres queridos cercanos, temor a la soledad y temor al futuro, que intentamos explicar a la luz del Análisis Existencial de Boss (1957), quien destaca la importancia de estar abiertos a las diferentes posibilidades del existir humano, aun cuando no podamos dominar aquellos procesos irreversibles como la muerte. 
No se han observado diferencias significativas en los puntajes obtenidos por estudiantes de acuerdo con variables sociodemográficas: edad, sexo, estado civil, Iglesia a la que pertenece, distrito en el que reside, colegio de procedencia, edad en que terminó el colegio, edad en que ingresó a la universidad, número de hermanos, lugar que ocupa entre los hermanos y si tiene o no un hobby, lo que nos hace pensar que la voluntad de sentido cualitativamente entendida (Frank1, 1988b) que manifiestan nuestros estudiantes, tiene también un caráter general con independencia de esas variables.

Lo que sí se puede resaltar es que existe un porcentaje elevado de alumnos cuyos padres tienen instrucción superior, un gran porcentaje trabaja en su profesión y un gran porcentaje de madres tienen una ocupación fuera de la casa, variables que podrían influir positivamente en la intensidad motivacional de búsqueda de metas noéticas de dichos jóvenes.

La intensidad motivacional de los alumnos de quinto ciclo se presenta como la más elevada en comparación con los estudiantes de los otros niveles de estudio, lo que evidencia que aún no tienen un sentido o propósito definido, por lo que su búsqueda es más intensa.

Se han observado diferencias significativas en los puntajes obtenidos por los hijos de padres que viven separados y los hijos de padres fallecidos en comparación con los alumnos que tienen padres que viven juntos. Esto puede ser interpretado como esa fuerza primaria de la que habla Frankl, que es la búsqueda por parte del hombre del sentido de la vida, y que de acuerdo con los resultados encontrados en la investigación no se ha producido una frustración existencial, sino que, por el contrario, existe un afán de encontrar un sentido concreto a la existencia personal o, lo que es lo mismo, la voluntad de sentido.

Los resultados cuantitativos (puntajes totales más bajos) y cualitativos (mayor claridad en los objetivos) hallados en el noveno ciclo y el internado son explicados en la logoterapia como pérdida de una tensión existencial necesaria entre el hombre y el sentido que ha de ser realizado por él, produciéndose una dis-tensión, que supone una vuelta o reflexión sobre sí mismo, para buscar un mayor sentido existencial a largo plazo.

De acuerdo con los resultados obtenidos en la prueba de Búsqueda de Metas Noéticas, cuya adaptación ha determinado validez, confiabilidad y validez de constructo, consideramos que sería importante continuar estas investigaciones en estudiantes universitarios de pregrado y posgrado de otras especialidades, teniendo en cuenta también otras variables sociodemográficas. 


\section{REFERENCIAS}

Aristóteles (1998). Ética Nicomaquea. México: Editorial Porrúa.

Acevedo, G. (1985). La búsqueda de sentido y su efecto terapéutico. Buenos Aires: Ediciones FAL.

Baltes, P. (1987). Theoretical propositions of life-span developmental psychology: On the dynamics between growth and decline. Developmental Psychology, 23, 611-623.

Biswanger, L. (1957) Sigmund Freud: Reminiscences of a friendship. Nueva York: Grune \& Stratton.

Boss, M. (1958). The analysis of dreams. Nueva York: Philosophical Library.

Boss, M. (1963) Psychoanalysis and Daseinsanalysis. Nueva York: Basis Books.

Corveleyn, J. (1998). Efectos psicológicos de la relación con pacientes en la persona del clínico. Persona, 1. Lima: Universidad de Lima.

Crumbaugh, J. (1968). Cross-validation of Purpose in Life Test based on Frankl's concepts. Journal of Individual Psychology. Vol. 24, pp. 74-81.

Crumbaugh, J. (1977). The seeking of Noetic Goals Test (SONG): A complementary scale to the Purpose in Life Test (PIL). Journal of Clinical Psychology. Vol. 33, 3, pp. 900-907.
Crumbaugh, J. \& Maholick, L. (1964). An experimental study in existentialism: The psychometric approach to Frankl's concept $f$ noogenic neurosis. Journal of Clinical Psychology. Vol. 20, 2, pp. 200-207.

Cuny, J. (2001). Actitud y sentimiento de soledad en adolescentes universitarios de Lima. Persona, 4. Lima: Universidad de Lima, pp. 111-128.

Ferrater Mora, J. (1969). Diccionario de filosofia. Buenos Aires: Sudamericana, tomo II, pp. 291-292.

Frankl, V. (2001). El hombre en busca de sentido. 21. ${ }^{\mathrm{a}}$ edición. Barcelona: Herder.

Frankl, V. (1998). El hombre en busca de sentido. 19. ${ }^{\mathrm{a}}$ edición. Barcelona: Herder.

Frankl, V. (1992). Teoría y terapia de la neurosis: Iniciación a la logoterapia y al análisis existencial. Barcelona: Herder.

Frankl, V. (1990). Logoterapia y análisis existencial: Textos de cinco décadas. Barcelona: Herder.

Frankl, V. (1988a). La presencia ignorada de Dios: Psicoterapia y religión. 7. ${ }^{\mathrm{a}}$ edición. Barcelona: Herder.

Frankl, V. (1988b). La voluntad de sentido. Conferencias escogidas sobre logoterapia. Barcelona: Herder. 
Frankl, V. (1987a). Ante el vacio existencial: Hacia una humanización de la psicoterapia. 5. ${ }^{\text {a }}$ edición. Barcelona: Herder.

Frank1, V. (1987b). El hombre doliente: Fundamentos antropológicos de la psicoterapia. Barcelona: Herder.

Frankl, V. (1970). Psicoanálisis y existencialismo. México: Fondo de Cultura Económica.

Guttman, D. (1998). Logoterapia para profesionales. Trabajo social significativo. Bilbao: Desclée De Brouwer.

Heidegger, M. (1950). Ser y tiempo. México: Colegio de México.

Hernández, S. et al. (1998). Metodología de la investigación. Bogotá: McGraw-Hill.

Jaspers, K. (1981). Filosofia desde el punto de vista de la existencia. Madrid: Fondo de Cultura Económica.

Jung, C. G. (1987). Psicología de la personalidad. México: Fondo de Cultura Económica.
Millán Puelles, Antonio. (2001). Fundamentos de filosofía. Madrid: Rialp.

Noblejas de La Flor, M. Ángeles (2000). Palabras para una vida con sentido. Bilbao: Desclée de Brouwer, Colección Serendipity.

Rychlak, J. (1981). Introduction to personality and psychotherapy. $2 .^{\mathrm{a}}$ edición. Boston: Houghton Mifflin Co.

Yalom, I. (1984). Psicoterapia existencial. Barcelona: Herder.

\section{Páginas web}

$<$ http://www.cgjungpage.org/>.

$<$ http://www.cgjungpage.org/articles/o brist1.html $>$.

$<$ http://aix1.uottawa.ca/ gwalters/kjsn a.html $>$.

$<$ http://www.philosophypages.com/dy/ j.htm>. 
Cuny 